\title{
Giant cell tumor stromal cells: Osteoblast lineage-derived cells secrete IL-6 and IL-10 for M2 macrophages polarization
}

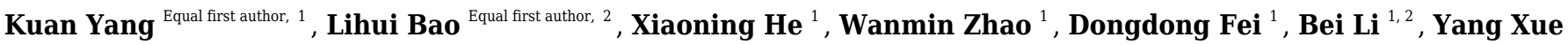 \\ Corresp., 3 , Zhiwei Dong ${ }^{\text {Corresp. } 1}$ \\ ${ }^{1}$ State Key Laboratory of Military Stomatology \& National Clinical Research Center for Oral Diseases \& Shaanxi International Joint Research Center for \\ Oral Diseases, Center for Tissue Engineering, School of Stomatology, Fourth Military Medical University, Xi'an, Shaanxi, China \\ 2 Xi'an Institute of Tissue Engineering and Regenerative Medicine, Xi'an, Shaanxi, China \\ 3 State Key Laboratory of Military Stomatology \& National Clinical Research Center for Oral Diseases \& Shaanxi Clinical Research Center for Oral Diseases, \\ Department of Oral and Maxillofacial Surgery, School of Stomatology, The Fourth Military Medical Unive, Xi'an, Shaanxi, China \\ Corresponding Authors: Yang Xue, Zhiwei Dong \\ Email address: xueyangfmmu@live.cn, narendzw1@163.com
}

Background. The giant cell tumor (GCT) is a benign tumor, which consists of three types cells: mononuclear histiocytic cells (MNHCs), multinuclear giant cells (MNGCs), and GCT stromal cells (GCTSCS). Numerous studies claim that GCTSCs have mesenchymal stem cells (MSCs) characters and play an important role in osteoclastogenesis, however, there are no research concerning about macrophage polarization among GCT, which can be regarded as an ingredient for tumor aggression. Method. We tested the effect of GCTSCS from three GCT samples which were collected from patients on proliferation, apoptosis and polarization of macrophage. Result. In this article, we verified that GCTSCs expressed MSCs markers and had higher proliferation and relative lower differentiation abilities compared with BMMSCs. What's more, we found a higher proportion of M2 macrophages among neoplasm. Co-culturing GCTSCs with macrophages resulted in prominent macrophage M2 polarization and increased the release of IL-6 (Interleukin-6) and IL-10 (Interleukin-10)from GCTSCs. In conclusion, GCTSCs, as originating from MSCs, can secret IL-6 and IL-10, which may play a significant role in macrophage M2 polarization. 
1 Giant Cell Tumor Stromal Cells: Osteoblast Lineage-Derived Cells Secret IL-6 and IL-10

2 for M2 Macrophages Polarization

3

4 Kuan Yang ${ }^{1 \#}$, Lihui $\mathrm{Bao}^{2 \#}$, Xiaoning $\mathrm{He}^{1}$, Wanmin Zhao ${ }^{1}$, Dongdong Fei ${ }^{1}$, Bei $\mathrm{Li}^{1,2}$, Yang Xue ${ }^{3 *}$, 5 Zhiwei Dong ${ }^{1 *}$

6

$7 \quad{ }^{1}$ State Key Laboratory of Military Stomatology \& National Clinical Research Center for Oral 8 Diseases \& Shaanxi International Joint Research Center for Oral Diseases, Center for Tissue 9 Engineering, School of Stomatology, Fourth Military Medical University, Xi'an, Shaanxi, China. 10 'Xi'an Institute of Tissue Engineering and Regenerative Medicine, Xi'an, Shaanxi, China.

$11{ }^{3}$ State Key Laboratory of Military Stomatology \& National Clinical Research Center for Oral 12 Diseases \& Shaanxi Clinical Research Center for Oral Diseases, Department of Oral and Maxillofacial Surgery, School of Stomatology, The Fourth Military Medical University, Xi'an, 14 Shaanxi, P. R. China

17 Corresponding Author:

18 Dr. Zhiwei Dong

19 narendzw1@163.com

20 Yang Xue

21 xueyangfmmu@live.cn 
27

28

29

30

31

32

33

34

35

36

37

38

39

40

41

42

43

44

45

46

47

48

49

50

51

52

53

54

55

56

57

\section{Abstract}

Background. The giant cell tumor (GCT) is a benign tumor, which consists of three types cells: mononuclear histiocytic cells (MNHCs), multinuclear giant cells (MNGCs), and GCT stromal cells (GCTSCs). Numerous studies claim that GCTSCs have mesenchymal stem cells (MSCs) characters and play an important role in osteoclastogenesis, however, there are no research concerning about macrophage polarization among GCT, which can be regarded as an ingredient for tumor aggression.

Method. We tested the effect of GCTSCs from three GCT samples which were collected from patients on proliferation, apoptosis and polarization of macrophage.

Result. In this article, we verified that GCTSCs expressed MSCs markers and had higher proliferation and relative lower differentiation abilities compared with BMMSCs. What's more, we found a higher proportion of M2 macrophages among neoplasm. Co-culturing GCTSCs with macrophages resulted in prominent macrophage M2 polarization and increased the release of IL6 (Interleukin-6) and IL-10 (Interleukin-10) from GCTSCs. In conclusion, GCTSCs, as originating from MSCs, can secret IL-6 and IL-10, which may play a significant role in M2 polarization of macrophages.

Key words: giant cell tumor stromal cells, macrophages, polarization, interleukin-6, interleukin10

\section{Introduction}

The giant cell tumor (GCT) is a locally aggressive benign tumor which incidence accounts for nearly 3-5\% of primary bone neoplasm [1]. About 80\% patients with GCT are aged 20 to 40. The GCT induced lesions usually occur at long bones, mostly located at distal femur, proximal tibia, and distal radius, sometimes at sacrum. The typical radiographic features of GCT include lytic mass with cystic degeneration and intact fibrous periosteum margin $[2,3]$.

GCT consists of three mainly cells types: the mononuclear histiocytic cells (MNHCs), which are driven from hematopoietic system, multinuclear giant cells (MNGCs), and GCT stromal cells (GCTSCs). GCTSCs are the neoplastic component of GCT, which is regarded as a promoter for accumulation, size and activity of giant cells [4-6]. It has been claimed that 
58 GCTSCs originate from mesenchymal stem cells (MSCs) [5, 7] and express MSCs markers 59 CD73, CD105, CD166 [7]. What's more, GCTSCs are also capable of multilineage

60 61

62

63

64

65

66

67

68

69

70

71

72

73

74

75

76

77

78

79

80

81

82

83

84

85

86

87

differentiation into adipocytes and osteocytes, which can secret bone matrix proteins such as type I collagen, osteocalcin and bone sialoprotein [7-10].

However, apart from the above features, the mechanisms that how GCTSCs affect tumor cell invasion and metastasis are still unclear. In view of GCT neoplasm is full filled with macrophages, we speculate GCTSCs may have a connection with tumor associated macrophages (TAMs) [11]. It has been evidenced that transplanting MSCs into experimental spinal cord injury model alternatively activated M2 macrophages [12]. As an anti-inflammatory factor, M2 macrophages regulate inflammatory responses and promote angiogenesis, tissue remodeling and repair, which enable them to play a significant role in tumor aggression [13, 14].

Some studies have demonstrated that MSCs can convert macrophages into M2 phenotype by producing IL-10 [15], which has been evidenced as major cytokine inducing M2 polarization [16-19]. In the current study, we analyzed the expression of cytokines in BMMSCs and GCTSCs. The result showed an enhanced secretion of IL-6 and IL-10 in GCTSCs while cocultured with macrophages. Considering GCTSCs originate from MSCs, it has been hypothesized that GCTSCs might influence M2 polarization of the macrophages in which IL-6 and IL-10 play an important role. In this study, we showed that GCTSCs promoted macrophages M2 polarization via increased IL-6 and IL-10 secreting, which resulted in GCT protruding aggression.

\section{Materials \& Methods}

Clinical samples

Three GCT tissue samples were collected from patients who underwent surgical resection for primary GCT in Department of Orthopaedics, Xijing Hospital, the Fourth Military Medical University. The patient's consent was obtained and informed consent form was signed. The use of all patient-derived materials was approved by the Ethics Committee of the Fourth Military Medical University (IRB-REV-2015005), and patient informed consent was obtained individually.

\section{Cell culture}

Peer) reviewing PDF | (2020:02:46214:1:2:NEW 14 Jul 2020) 
88 Primary GCT stromal cells (GCTSCs) were isolated from fresh GCT. The neoplasm was minced 89 in small pieces and digested with $200 \mathrm{U} / \mathrm{ml}$ collagenase (Gibco BRL, Karlsruhe, Germany) for 1 $90 \mathrm{~h}$ at $37^{\circ} \mathrm{C}$. Cells were collected by centrifugation, washed twice in PBS and cultured with alpha 91 minimum essential medium ( $\alpha$-MEM, Gibco BRL, Gaithersburg, MD, USA) containing 10\% 92 FBS (Hangzhou Sijiqing Biological Engineering Materials Co., Ltd. Zhejiang, China) and 100 $93 \mathrm{U} / \mathrm{ml}$ penicillin and $100 \mathrm{mg} / \mathrm{ml}$ streptomycin (Invitrogen Life Technology, Carlsbad, CA, USA). 94 The cells were kept in culture at $37^{\circ} \mathrm{C}, 5 \% \mathrm{CO}_{2}$, and the media was changed every 3 days 95 thereafter until cells reached 70\% confluency. Adherent GCTSCs were harvested using $0.25 \%$ 96 Trypsin-EDTA (Invitrogen Life Technology, Carlsbad, CA, USA). Normal MSCs were obtained 97 from the bone marrow of the normal donor patients as described previously ( Soleimani M and 98 Nadri S 2009). Human monocytes were isolated from the peripheral blood of normal human 99 volunteers (Blood donors in Blood Transfusion Department of Xijing Hospital) using human 100 monocyte isolation kit II (Miltenyi Biotech, Teterow, Germany). Purified CD14 monocytes 101 were plated into 6-well cell culture plates at a concentration of $1 \times 10^{6}$ per well in RPMI 1640 102 media supplemented with $10 \%$ FBS.

103 Animal experiments

104 Immunocompromised mice (NMRI-nu/nu) were purchased from Beijing Vital River Laboratory, 105 Beijing, China. All mice were housed five per cage under standard laboratory conditions at $12 \mathrm{~h}$ 106 light /dark cycles, and $22^{\circ} \mathrm{C}$, with free access to food and water. Mice were anesthetized by 107 intraperitoneal injection of pentobarbital $(30 \mathrm{mg} / \mathrm{kg})$ and implanted with cells. After 35 days of 108 implantation, the mice were decapitated and killed, the de novo bone area was taken. Animal 109 experiments in this study was authorized by the Animal Care Committee of Fourth Military 110 Medical University (2016-kq-054), and all experimental protocols were performed with the 111 approval of the Fourth Military Medical University.

112 Histology and Immunohistochemistry Staining

113 The neoplasm specimens were fixed in 4\% paraformaldehyde overnight and then embedded in 114 paraffin or in the optimal cutting temperature compound. Hematoxylin and eosin (HE) staining 115 was used for general observation. Surface markers of GCTSCs and macrophages were stained 116 with CD105 and CD14 antibodies (eBiosciences, San Diego, CA, USA) overnight at $4^{\circ} \mathrm{C}$, 117 respectively. When they were washed 3 times, peroxidase conjugated goat anti-rabbit secondary 118 antibody (Dako, Denmark) was added. Envision Detection System kit (DAKO, Denmark) was 
119 used after the nuclear stained by hematoxylin. Color development was triggered using Di-Amino 120 Benzidine (DAB, Calbiochem, Germany).

121 Immunofluorescent Staining

122 BMMSCs and GCTSCs were washed with PBS and fixed with 4\% paraformaldehyde for 15 min, 123 and then were incubated with antibodies CD146, CD90, CD73, STRO-1 and SSEA-4, as well as 124 antibodies against hematopoietic cell markers of CD14 and CD34 and CD45 (A1l from BD 125 Biosciences, San Diego, CA, USA). The positively stained cells were detected by a laser 126 scanning confocal microscope (Olympus FluoView FV 1000, Tokyo, Japan).

\section{Flow Cytometry Analysis}

128 To confirm mesenchymal stem cells character, we used flow cytometric analysis to show that 129 GCTSCs were positive for CD90, CD105, and CD29, and negative for CD14, CD34 and CD45 130 (All from BD Biosciences, San Diego, CA, USA). Ki-67 staining was used to confirm the 131 proliferation of macrophages after being co-cultured with GCTSCs. Apoptotic cells were 132 examined using Annexin V-FITC/7AAD Apoptosis Detection Kit (Roche, Basel, Switzerland). 133 Cells were stained with Annexin V-FITC and 7AAD for $15 \mathrm{~min}$, both of which were in the dark 134 at room temperature. After washing by PBS, immunofluorescent-stained cells were analyzed on 135 FACS (Becton-Dickinson) can using Consort 30 software.

\section{BrdU test}

137 BMMSCs and GCTSCs were labeled with 5-bromodeoxyuridine (BrdU, sigma) $10 \mathrm{umol} \cdot \mathrm{L}^{-1}$ for $13824 \mathrm{~h}$. After the cells were washed three times with PBS and fixed with 4\% paraformaldehyde for $13915 \mathrm{~min}$, the incorporated BrdU was detected by mouse anti-BrdU monoclonal antibody 140 conjugated with peroxidase (Abcam). The color reaction was parallel with the number of 141 proliferating cells.

\section{Osteogenic and Adipogenic Differentiation}

143 BMMSCs and GCTSCs were incubated with osteogenic medium $\left(100 \mathrm{nmol} \cdot \mathrm{L}^{-1}\right.$ dexamethasone,

$14450 \mathrm{mg} \cdot \mathrm{mL}^{-1}$ ascorbic acid and $1 \mathrm{mmol} \cdot \mathrm{L}^{-1} \mathrm{~b}$-glycerophosphate, Sigma) for $21 \mathrm{~d}$ according to the 145 manufacturer's instructions. Cells were fixed with $60 \%$ isopropanol and stained with $1 \%$ 146 Alizarin Red (Sigma) for osteogenic differentiation assay. BMMSCs and GCTSCs were also 147 cultured with adipogenic medium $\left(0.5 \mathrm{mmol} \cdot \mathrm{L}^{-1}\right.$ methylisobutylxanthine, $0.5 \mathrm{mmol} \cdot \mathrm{L}^{-1}$ 148 hydrocortisone and $60 \mathrm{mmol} \cdot \mathrm{L}^{-1}$ indomethacin, Sigma) for $14 \mathrm{~d}$. Intracellular lipid droplet was 149 detected by staining with Oil Red O solution. 


\section{In vivo bone formation assay}

$1515 \times 10^{6}$ cells were combined with $40 \mathrm{mg}$ hydroxyapatite/tricalcium phosphate (HA/TCP) and 152 implanted at the back of immunocompromised mice (NMRI-nu/nu). After 35 days the mice were 153 euthanized and the de novo bone area was stained by HE. The sections were analyzed by Image154 Pro Plus software.

155 Co-cultures

156 The purified human blood monocytes were indirectly co-cultured with GCTSCs as well as 157 BMMSCs for $14 \mathrm{~d}$. The monocytes $\left(2 \times 10^{5}\right.$ cells $)$ were cultured in 24 well plates and GCTSCs or 158 BMMSCs $\left(2 \times 10^{4}\right.$ cells) were cultured in the cell-culture insert (pore size: $\left.0.4 \mathrm{pm}\right)$ of the same 159 well (Falcon Inc.).

160 Antibody-based protein array

161 The assay was performed following the manufacturer's instructions (RayBio). Briefly, samples 162 with equal amounts of total proteins were incubated overnight at $4^{\circ} \mathrm{C}$. After washing, the 163 membranes were incubated with Biotinylated Antibody Cocktail followed by second wash. 164 Incubate with HRP-Streptavidin overnight at $4^{\circ} \mathrm{C}$ and wash again. Chemiluminescence was used 165 for signal detection, which was analyzed with Quantity One image analysis software (Molecular 166 Imager FX, Bio-Rad Laboratories).

167 Quantitative RT-PCR

168 Total RNA was isolated from BMMSCs and GCTSCs by using RNAiso plus (TaKaRa, Tokyo, 169 Japan) according to the manufacturer's instructions. The mRNA was reversed to complementary 170 DNA, and quantitative real time -PCR (qRT-PCR) detection was carried out by Prime Script TM 171 RT master mix (TaKaRa, RR036A) and SYBR Premix Ex TaqTMII (TaKaRa). A CFX96 172 Trademark Real-time PCR detection system (Bio-Rad, Richmond, CA, USA) was used for the 173 detection. The forward and reverse primers were used to measure IL-6 (F: 174 AgtgagGaACAAGCCAGAGC,R: AGCTGCGCAGAATGAGATGA) and IL-10 (F: 175 ACCTGCCTAACATGCTTCGAG, R: TGGGTCTTGGTTCTCAGCTTG) gene expression, and 176 target gene expression levels were normalized to that of GAPDH (F: 177 TGACATCAAGAAGGTGGTGAAGC, R: GGAAGAATGGGAGTTGCTGTTG).

\section{Gene ablation by siRNA}

$1792 \times 10^{5}$ cells / well were seeded in a 6-well culture plate with $2 \mathrm{ml}$ antibiotic- free normal growth 180 medium supplemented with FBS. Incubated the cells at $37^{\circ} \mathrm{C}$ in a $\mathrm{CO} 2$ incubator until the cells 
181 were $60-80 \%$ confluent. Then added IL-6 (Santa) and IL-10 (Santa) siRNA duplex and siRNA

182

183

184

185

186

187

188

189

190

191

192

193

194

195

196

197

198

199

200

201

202

203

204

205

206

207

208

209

210

211

transfection reagent (Santa) mixture after cells were washed with $2 \mathrm{~mL}$ siRNA transfection medium, then incubate the cells 5-7 hours at $37^{\circ} \mathrm{C}$ in a $\mathrm{CO}_{2}$ incubator. $1 \mathrm{~mL}$ of medium containing 2 times FBS and antibiotics was added to cells and incubated for additional 18-24 hours before used.

\section{Statistical Analysis}

GraphPad Prism software (Graph-Pad Software, Inc., USA) was used for statistical analysis. All data are presented as mean \pm s.d and the measurements were performed on three different GCT patient samples $(\mathrm{n}=3)$. Unpaired, two-tailed Student's $\mathrm{t}$ tests for comparisons between two groups and one-way analysis of variance (ANOVA) with Bonferroni for multiple comparisons were applied. Each experiment was repeated at least three times. P value $<0.05$ was considered to be statistically significant.

\section{Results}

\section{CD14 and CD105 cells exist in Gaint cell tumor}

We harvested Giant cell tumor (GCT) samples from three patients with two females (46 and 41 years old) and one male (21 years old). The lesions in which were tibia, ilium and both terminal of the tibia and femur. All of these three patients presented a common radiologic feature: a lytic and well-defined lesion without sclerotic margin (Figure 1a-c). HE staining showed GCT consisted of three kinds of cells: multinucleated giant cells, mononuclear histocytic cells and giant cell tumor stromal cells (GCTSCs) with a spindle morphology. Immunohistochemistry staining verified that GCT cells contained CD14 positive and CD105 positive cells, which represented a macrophage-like and mesenchymal-like features respectively (Figure 1d). Immunofluorescence staining of the GCT indicated STRO-1 positive and CD14 positive cells in the samples and these two kinds of cells were not overlapped (Figure 1e, f).

\section{GCTSCs showed increased proliferation and decreased differentiation characteristics}

It had been widely proved that giant cell tumor stromal cells (GCTSCs) were the neoplastic component of GCT and had similar functions with mesenchymal stem cells. We isolated the cells from neoplasm and compared different bio-markers (CD90, CD105, CD29, CD14, CD34, CD45) between three patients' GCTSCs and normal bone marrow MSCs. Flow cytometer analysis 
212 indicated that both GCTSCs and BMMSCs had a similar specific expression of CD90, CD105 213 and CD29 and negative expression of CD14, CD34 or CD45 (Figure 2a). To characterize the 214 proliferation property of GCTSCs, we performed serial implantation of GCTSCs. Brdu 215 measurement indicated that all of three GCTSCs had a significantly higher proportion of Brdu216 positive cells than BMMSCs $(\mathrm{P}<0.01)$. After subcutaneously implantation of GCTSCs into 217 immunocompromised mice, we found that re-GCTSCs collected from implants still showed 218 higher proliferation than re-BMMSCs. (Figure 2b, c). These results indicated GCTSCs had 219 mesenchymal stem cell like properties and had an obviously higher proliferative activity than 220 BMMSCs.

221 In order to examine the differentiative capacity of GCTSCs into osteoblast and adipocyte, 222 we induced the GCTSCs with osteogenic and adipogenic induction medium respectively. 223 Alizarin Red staining verified that the total mineralized area in GCTSCs was 0.11 compared with 2240.25 in BMMSCs (2.27-fold higher, $\mathrm{P}=0.056)$ (Figure 2d). Oil-Red $\mathrm{O}$ stained cells represented 225 the adipocytes, the proportion of which in GCTSCs was $2.22 \%$ compared with $32.44 \%$ in 226 BMMSCs (14.61-fold higher, $\mathrm{P}<0.01)$ (Figure 2e). GCTSCs or BMMSCs combined HA/TCP 227 were subcutaneously implanted into immunocompromised mice, and a clearly bone formation 228 area of BMMSC implantation was founded, the total de novo bone area was about $0.12 \%$, 229 compared with much smaller spots about 0.04\% among GCTSC implantation $(\mathrm{P}<0.001)$ (Figure 230 2f). These results indicated that GCTSCs indeed had bone and adipose differentiation capacities 231 to some extent in vitro as well as in vivo, but which were much weaker than BMMSCs.

232

233

GCTSCs play no role in proliferation or apoptosis of CD14 cells

234

235

Many researches had pronounced that GCTSCs played a significant role in giant cells formation, osteolysis as well as aggression, but there were rarely researches to explore the 236 influence of GCTSCs on the proliferation or apoptosis of CD14 cell. Co-culturing macrophage 237 cells with GCTSCs and BMMSCs using transwell for 7 days, 14 days and 21 days respectively 238 and the amount of macrophage cells was counted. The results of different co-culturing period 239 with GCTSCs or BMMSCs exhibited no significance correlation (Figure 3a). The proportion of

$240 \mathrm{Ki} 67$ cells, as an indication of proliferative ability, among normal macrophages and co-cultured 241 with BMMSCs as well as GCTSCs are 3.83\%, 2.57\% and 4.72\% (P>0.05) (Figure 3b, d), which 
242 indicated both GCTSCs and BMMSCs didn't alter the proliferative ability of CD 14 positive 243 macrophages.

244 Moreover, we detected the Annexin V+7-ADD+ macrophages after co-cultured with 245 BMMSCs or GCTSCs. BMMSCs had a prominent tendency in inducing macrophage apoptosis, 246 the proportion of which was approximately 3.65\% $(\mathrm{P}=0.01)$, however, co-culture with GCTSCs 247 hadn't resulted in any enhancement of macrophage apoptosis, which were $1.25 \%, 3.65 \%$ and $1 \%$ 248 (BMMSCs vs Macrophage, $\mathrm{P}<0.05$. GCTSCs vs Macrophage, $\mathrm{P}>0.05$ ), respectively (Figure 3c, 249 d). These data confirmed that BMMSCs could induce the apoptosis of macrophage but not the 250 GCTSCs. However, the proportion of apoptosis cells induced by BMMSCs was very low.

251

252 253

GCTSCs promote the polarization into M2 phenotype of macrophage via increasing IL-6 and $\mathrm{IL}-10$ release

254

255

It had been wildly accepted the polarization of macrophage into M2 phenotype was a contribution for tumor propagation and angiogenesis. To investigate the polarization of 256 macrophages in GCT, we detected the proportion of M2 phenotype macrophages in GCT and 257 peripheral blood respectively, we found a numerous proportion of M2 phenotype macrophages 258 among the neoplasm $(85.05 \%)$ compared with normal macrophages $(61.15 \%)(\mathrm{P}<0.05)$. This suggests that a high number of M2 macrophages exist in giant cell tumor of bone. (Figure 4a). 260 By co-culture the macrophages with GCTSCs as well as BMMSCs, it aroused a significant 261 $(11.0 \%, 11.6 \%, 19.1 \%)$ polarization of M2 macrophages in GCTSCs and 8.6\% in BMMSCs (Figure 4b). These results verified that, compared with BMMSCs, GCTSCs could promote the 263 macrophages into M2 phenotype.

264

Human cytokine antibody array can semi-quantitative detected 42 human proteins 265 expression using 2-D densitometry. By analyzing the culture supernatant of BMMSCs and 266 GCTSCs, it was clarified that GCTSCs had a distinctive extraction in the spot of IL-6 and IL-10 (Figure 4c). q-PCR analysis verified that GCTSCs had a much higher expression of IL-6 and IL10 compared with BMMSCs $(\mathrm{P}<0.05)$ (Figure $4 \mathbf{d})$. In order to verify the promotion effect of 269 increased GCTSCs IL-6/10 expression on GCT macrophages M2 activation, we used siRNA to reduce the expression of IL-6/10 in GCTSCs, and then co-cultured with THP-1 cells for 48

271 hours. The results of cell flow cytometry showed that knock down the expression of IL-6/10 in 272 GCTSCs could reduce the proportion of THP-1 CD206 positive cells in the co-culture system 
$273(\mathrm{P}<0.05)$ (Figure 4e). These results illustrated that GCTSCs expressed and secreted a large 274 amount of IL-6 and IL-10, which might induce the polarization into M2 phenotype macrophages.

275

276

\section{Discussion}

278

279

280

281

282

283

284

285

286

287

288

289

290

291

292

293

294

295

296

297

298

299

300

301

302

It has been wildly accepted that GCTSCs are the neoplasms component of the bone and express mesenchymal stem cell (MSCs) markers $[5,7,9]$. In this study, we confirm GCTSCs express MSCs-like markers STRO-1, SSEA-4, CD90, CD146, CD105, CD29, which are in accordance with BMMSCs $[9,20]$, and is the neoplastic component, which have a prominent proliferative activity that can be stained in Brdu measurement. Others reported that GCTSCs expressed alkaline phosphatase, osteocalcin and Cbfa1, equipping a multi-differentiation ability to form mineralized node and adipose not only in vitro, but also in vivo [10, 21]. In the present results, GCTSCs could differentiate into osteoblasts and adipocytes, which was in accordance with the previous findings. However, comparing with the BMMSCs, GCTSCs have weaker differentiation capacities.

Previous researches had reported that tumor cells could recruiting circulating monocytes, osteoclast precursor cell or even osteoclasts by producing transforming growth factor-1 (TGF$\beta 1$ ) and monocyte chemoattractant protein 1 (MCP-1) [22]. Jenkins SJ et al discovered that IL-4 and filarial nematode infection can induce resident macrophages proliferation without recruiting blood cells [23]. In order to confirm if GCTSCs can alter macrophages proliferative ability, we co-cultured macrophage cells with GCTSCs and BMMSCs using transwell. The Ki67 cells exhibit a similar proportion among control, GCTSCs and BMMSCs groups, which means GCTSCs don't induce macrophages proliferation. Simultaneously, the proportion of Annexin $\mathrm{V}+7 \mathrm{ADD}+$ macrophages was increased after co-culture with BMMSCs, indicating that BMMSCs can induce more macrophage cells apoptosis than GCTSCs.

Nevertheless, no former research has focused on the relationship between GCTSCs and macrophages polarization. We for the first time discovered a numerous proportion of M2 phenotype macrophage cells among the neoplasm (85.05\%) compared with normal macrophages $(63.15 \%)(\mathrm{P}<0.05)$. Then, we co-cultured GCTSCs with macrophages and it exhibited a higher proportion of M2 macrophages than BMMSCs. By analyzing the culture supernatant of BMMSCs and GCTSCs with human cytokine antibody array, we found a higher IL-6 and IL-10 
304 secretion, consistent with the array data, an increased mRNA expression was found in IL-6 and 305 IL-10. Interestingly, knockdown of the IL-6 and IL-10 expression in GCTSCs cells with siRNA 306 attenuated its effect on THP-1 cells M2 polarization. Therefore, we conclude that the cytokines 307 secreted by GCTSCs may engage in macrophage M2 polarization.

308 It has been clarified that MSCs can alter the phenotype of macrophages from M1 type into a 309 M2 population, which are indicative of pro-tumor activity [14, 24], but there are no articles that 310 analyze macrophage phenotype among GCT. Former researchers had claimed that 311 immunoregulation cytokines like IL-4, IL-10 and IL-13 induced the M2 phenotype polarization 312 [19]. However fewer articles reported that IL-6 may also be a novel cytokine that plays an 313 important role in M2 macrophage polarization [18, 25]. In our research, IL-6 is obviously 314 involved in the polarization of macrophages M2, but the mechanism still needs more exploration. 315 STAT3 is an important mechanism for inducing the activation of tumor-associated macrophages 316 M2 type [26]. It has been reported that in the tumor microenvironment, the IL-6/STAT3 317 signaling pathway plays an important role in the macrophage M2 polarization, and activation of 318 the IL-6/STAT3 signaling pathway causes macrophages to polarize towards the M2 phenotype. 319 Inhibiting the IL-6/STAT3 signaling pathway correspondingly shifts macrophages to the M1 320 polarization phenotype, and blocking the IL-6/STAT3 signaling pathway can dramatically 321 reduce tumor formation [26]. Further, IL-6 binds to the IL-6 receptor subunit $\alpha$ (IL-6RA) and the 322 co-receptor gp130 to activate JAK and signal transduction and transcription activator 3 (STAT3) 323 phosphorylation, thereby activating the inflammatory cascade [26]. In addition, IL-10 can induce 324 IL-4R $\alpha$ expression and increase Arg1 expression, the one of markers of macrophage M2 325 activation. It was also be reported that IL-10 can enhance the expression of M2a macrophage326 related genes in M2 macrophages induced by IL-4 in vitro [27]. As we have detected both IL-6 327 and IL-10 high secretion of GCTSCs, it's interested for us to explore the connection between 328 GCTSCs and M2 macrophages. In this article, there are still many phenomena need further 329 explore. (a) Whether the M2 polarization on account of GCTSCs play a significant role in GCT 330 invasion and metastasis. (b) The exact mechanisms of IL-6 and IL-10 in inducing M2 331 polarization among GCT are still unknown. In conclusion, we first confirm that GCTSCs are 332 belong to osteoblast lineage and have an enhanced proliferative ability as well as a deduced 333 differentiative ability. Although numerous populations of macrophages emerge in giant cell 334 tumor, GCTSCs have none influence on its proliferation of apoptosis. IL-6 and IL-10 are found 
335

336

337

338

339

340

341

\section{2}

343

344

345

346

347

348

349

350

351

352

353

354

355

356

357

358

359

360

361

362

363

364

365

expressed highly among GCTSCs, while they are speculated to play a significant role in M2 phenotype macrophages. Our findings are consistent with previous reports that high level of M2 phenotype activation of tumor-associated macrophages in tumor microenvironment. In particular, we found that the increased expression of GCTSCs IL-6 and IL-10 is the key factor that causes the activation of M2 of macrophages. This provides a novel prospect for the clinical treatment of giant cell tumors of bone.

\section{Conclusions}

Our research proves that, GCTSCs expressed MSCs markers and had higher proliferation and relative lower differentiation abilities compared with BMMSCs. Co-culturing GCTSCs with macrophages promoting macrophages polarization into M2 phenotype (Figure 5). At the same time, the level of IL-6 and IL-10 released from GCTSCs was increased. In short GCTSCs contribution for tumor proliferation via promoting macrophages M2 phenotype polarization by improving IL-6 and IL-10 secretion (Figure 5).

Figure 1. CD14 and CD105 cells are observed in Gaint cell tumor by tissue staining. (a-c) X-Ray indicates the lesion area of three GCT patients are tibia, ilium and both terminal of the tibia and femur, respectively. (d) HE staining indicates that the neoplasm is consist of three categories cells: multinucleated giant cells, mononuclear histiocytic cells and GCTSCs. Scale bar $=50 \mu \mathrm{m}$. (e-g) Immunofluorescent staining exhibits the STRO-1 ${ }^{+}$cells (green) have a different distribution with $\mathrm{CD} 14^{+}$cells (red), which means that they belong to two distinguished lineages. Scale bar $=10 \mu \mathrm{m}$.

\section{Figure 2. GCTSCs showed increased proliferation and decreased differentiation} characteristics. (a) Flow cytometry analysis indicates that both of BMMSCs and GCTSC1, GCTSC2, GCTSC3 have common expressed MSCs markers CD90, CD105, CD29, and rarely express hematopoietic lineage markers CD14, CD34, CD45. (b, c) Proliferation ability of both BMMSCs and GCTSCs. Brdu measurement indicates the proliferating cells. Single-colonyderived MSCs and GCTSCs, respectively, were expanded to $2 \times 10^{6}$ cells and subsequently implanted into immunocompromised mice with the use of geofoams as a carrier. The proliferating cells in GCTSCs1 $(\mathrm{P}<0.01)$ and GCTSCs2 $(\mathrm{P}<0.05)$ are much more than BMMSCs. 
366 However, the GCTSCs3 after implantation shows a similar proliferation ability. (d, e)

367 Osteogenic and adipogenic differentiation of BMMSCs and GCTSCs in vitro. (f) de novo bone 368 formation of BMMSCs and GCTSCs in vivo.

369

370 Figure 3. GCTSCs play no role in proliferation or apoptosis of CD14 cells. (a) The 371 macrophages number co-cultured with BMMSCs and GCTSCs were compared in 7, 14 and 21 372 days. It shows that the both BMMSCs and GCTSCs have a rare effect on macrophages cells 373 number. (b) Ki67 expression in macrophages co-culturing with different types of cells shows a 374 similar level $(\mathrm{P}>0.05)$, which indicates that both BMMSCs and GCTSCs don't have a promotion 375 for macrophages proliferation. (c) The apoptosis of macrophages co-culturing with different 376 types of cells shows that BMMSCs $(\mathrm{P}<0.001)$ have a prominent tendence to induce macrophages 377 apoptosis, compared with GCTSCs $(\mathrm{P}>0.05)$. (d) Representative flow cytometry analysis of

378

379

380

381

382

383

384

385

386

387

388

389

390

391

392

393

394

Figure 4. GCTSCs promote the polarization into M2 phenotype of macrophage via increasing IL-6 and IL-10 release. (a) The proportion of CD206 ${ }^{+}$cells among CD14 ${ }^{+}$cells. CD206 is a marker of M2 macrophages and it consists $85.05 \%$ of macrophages in GCT compared with $61.15 \%$ in blood $(\mathrm{P}<0.05)$. (b) $\mathrm{CD}^{206^{+}}$cells proportion in $\mathrm{GCT} \mathrm{CD} 14^{+}$cells and co-cultured with BMMSC, GCTSC1, GCTSC2 and GCTSC3 (GCT CD14 ${ }^{+}$cell vs. GCT CD14 cell + BMMSCs, $\mathrm{P}=0.483$. GCT $\mathrm{CD} 14^{+}$cell vs. GCT CD $14^{+}$cell + GCTSCs $1, \mathrm{P}=0.026$. GCT $\mathrm{CD} 14^{+}$cell vs. GCT $\mathrm{CD}_{14}{ }^{+}$cell $+\mathrm{GCTSCs} 2, \mathrm{P}=0.044$. GCT CD14 ${ }^{+}$cell vs. GCT CD14 ${ }^{+}$cell +GCTSCs3, P<0.001). (c) Human cytokine antibody array analysis of BMMSCs and GCTSCs. Three independent experiments obtained a similar result and one of them are shown. The expression of IL-6 and IL-10 of GCTSCs is much higher compared with BMMSCs (POS=Positive Control Spot, $\mathrm{NEG=Negative} \mathrm{Control} \mathrm{Spot,} \mathrm{BLANK=Blank} \mathrm{Spot).} \mathrm{(d)} \mathrm{mRNA}$ expression level of IL-6 and IL-10 among BMMSCs and GCTSCs. The expression of IL-6 and IL-10 of GCTSCs is much higher compared with BMMSCs $\left({ }^{*} \mathrm{P}<0.05,{ }^{* * *} \mathrm{P}<0.001\right)$. (e) The proportion of CD206 ${ }^{+}$cells among THP-1 cells.

Peer] reviewing PDF | (2020:02:46214:1:2:NEW 14 Jul 2020) 
395 Figure 5. Schematic diagram of this study. Schematic illustration of GCTSCs exist in giant

396

397 phenotype.

\section{Compliance with Ethical Statements}

400 Conflict of Interest The authors declare that they have no conflicts of interest.

401 Funding This work was supported by the grants from the National Key Research and 402 Development Program of China (Nos. 2016YFC1101400), the National Natural Science 403 Foundation of China (Nos. 81870768 and 881991504) and the Guangzhou Science, Technology and Innovation Commission (201803040008, 201704030024).

Informed consent Informed consent was obtained from all individual participants included in the study.

407

408

409

410

411

412

413

414

415

416

417

418

419

420

421

422

423

424

425

426

427

428

429

430

431

432

433

434

435

436

437

438

439

\section{References}

[1] D.M. Thomas, K.M. Skubitz, Giant cell tumour of bone, Current Opinion in Oncology, 21 (2009) 338-344.

[2] K.A. Raskin, J.H. Schwab, H.J. Mankin, D.S. Springfield, F.J. Hornicek, Giant cell tumor of bone, J Am Acad Orthop Surg, 21 (2013) 118-126.

[3] Chakarun CJ, Forrester DM, Gottsegen CJ, Patel DB, White EA, Matcuk GR Jr. Giant cell tumor of bone: review, mimics, and new developments in treatment. Radiographics. 2013;33(1):197-211. doi:10.1148/rg.331125089

[4] M. Wuelling, G. Delling, E. Kaiser, Differential gene expression in stromal cells of human giant cell tumor of bone, Virchows Arch, 445 (2004) 621-630.

[5] M. Werner, Giant cell tumour of bone: morphological, biological and histogenetical aspects, Int Orthop, 30 (2006) 484-489.

[6] M.H. Zheng, P. Robbins, J. Xu, L. Huang, D.J. Wood, J.M. Papadimitriou, The histogenesis of giant cell tumour of bone: a model of interaction between neoplastic cells and osteoclasts, Histol Histopathol, 16 (2001) 297-307.

[7] P. MANUELA WU“" LLING, GUENTER DELLING, MD,AND EDELGARD KAISER, PHD, The Origin of the Neoplastic Stromal Cell in Giant Cell Tumor of Bone, (2003).

[8] D. Robinson, M. Segal, Z. Nevo, Giant cell tumor of bone. The role of fibroblast growth factor 3 positive mesenchymal stem cells in its pathogenesis, Pathobiology, 70 (2002) 333-342.

[9] J. Lan, X. Liu, W. Rong, F. Wei, L. Jiang, H. Yu, G. Dang, Z. Liu, Stro-1(+) stromal cells have stem-like features in giant cell tumor of bone, J Surg Oncol, 106 (2012) 826-836.

[10] A. Murata, T. Fujita, N. Kawahara, H. Tsuchiya, K. Tomita, Osteoblast lineage properties in giant cell tumors of bone, J Orthop Sci, 10 (2005) 581-588.

[11] J. Kim, J.S. Bae, Tumor-Associated Macrophages and Neutrophils in Tumor Microenvironment, Mediators Inflamm, 2016 (2016) 6058147.

[12] H. Nakajima, K. Uchida, A.R. Guerrero, S. Watanabe, D. Sugita, N. Takeura, A. Yoshida, G. Long, K.T. Wright, W.E. Johnson, H. Baba, Transplantation of mesenchymal stem cells promotes an alternative pathway of macrophage activation and functional recovery after spinal cord injury, J Neurotrauma, 29 (2012) 1614-1625.

[13] A. Mantovani, S. Sozzani, M. Locati, P. Allavena, A. Sica, Macrophage polarization: tumor-associated macrophages as a paradigm for polarized M2 mononuclear phagocytes, Trends Immunol, 23 (2002) 549-555.

[14] R. Ohashi, K. Yanagihara, S. Namimatsu, T. Sakatani, H. Takei, Z. Naito, A. Shimizu, Osteoclast-like giant cells in invasive breast cancer predominantly possess M2-macrophage phenotype, Pathol Res Pract, 214 (2018) 253258.

[15] Hutchins AP, Poulain S, Miranda-Saavedra D. Genome-wide analysis of STAT3 binding in vivo predicts

Peer] reviewing PDF | (2020:02:46214:1:2:NEW 14 Jul 2020) 
440 effectors of the anti-inflammatory response in macrophages. Blood. 2012;119(13):e110-e119. doi:10.1182/blood441 2011-09-381483

442 [16] P.J. Murray, Macrophage Polarization, Annu Rev Physiol, 79 (2017) 541-566.

443 [17] J. Jiang, T. Jia, W. Gong, B. Ning, P.H. Wooley, S.Y. Yang, Macrophage Polarization in IL-10 Treatment of 444 Particle-Induced Inflammation and Osteolysis, Am J Pathol, 186 (2016) 57-66.

445 [18] J. Mauer, B. Chaurasia, J. Goldau, M.C. Vogt, J. Ruud, K.D. Nguyen, S. Theurich, A.C. Hausen, J. Schmitz, 446 H.S. Bronneke, E. Estevez, T.L. Allen, A. Mesaros, L. Partridge, M.A. Febbraio, A. Chawla, F.T. Wunderlich, J.C. 447 Bruning, Signaling by IL-6 promotes alternative activation of macrophages to limit endotoxemia and obesity448 associated resistance to insulin, Nat Immunol, 15 (2014) 423-430.

449 [19] K.K. Goswami, T. Ghosh, S. Ghosh, M. Sarkar, A. Bose, R. Baral, Tumor promoting role of anti-tumor 450 macrophages in tumor microenvironment, Cell Immunol, 316 (2017) 1-10.

[20] X. ZC, D. J, L.T.J. Histology, histopathology, Commonly used mesenchymal stem cell markers and tracking labels: Limitations and challenges. A Lin CS, 28 (2013) 1109-1116.

[21] M. Nishimura, K. Yuasa, K. Mori, N. Miyamoto, M. Ito, M. Tsurudome, M. Nishio, M. Kawano, H. Komada, A. Uchida, Y. Ito, Cytological properties of stromal cells derived from giant cell tumor of bone (GCTSC) which can induce osteoclast formation of human blood monocytes without cell to cell contact, J Orthop Res, 23 (2005) 979-987. [22] L. Huang, J. Xu, D.J. Wood, M.H. Zheng, Gene expression of osteoprotegerin ligand, osteoprotegerin, and receptor activator of NF-kappaB in giant cell tumor of bone: possible involvement in tumor cell-induced osteoclastlike cell formation, Am J Pathol, 156 (2000) 761-767.

[23] Jenkins SJ, Ruckerl D, Cook PC, Jones LH, Finkelman FD, Rooijen NV, Macdonald AS, Allen JE. Local macrophage proliferation, rather than recruitment from the blood, is a signature of TH2 inflammation. Science. 2011;332(6035):1284-1288.

[24] M. Hajkova, E. Javorkova, A. Zajicova, P. Trosan, V. Holan, M. Krulova, A local application of mesenchymal stem cells and cyclosporine A attenuates immune response by a switch in macrophage phenotype, J Tissue Eng Regen Med, 11 (2017) 1456-1465.

[25] X.L. Fu, W. Duan, C.Y. Su, F.Y. Mao, Y.P. Lv, Y.S. Teng, P.W. Yu, Y. Zhuang, Y.L. Zhao, Interleukin 6 induces M2 macrophage differentiation by STAT3 activation that correlates with gastric cancer progression, Cancer Immunol Immunother, 66 (2017) 1597-1608.

[26] Yin Z, Ma T, Lin Y, Lu X, Zhang CZ, Chen S, Jian ZX. IL-6/STAT3 pathway intermediates M1/M2 macrophage polarization during the development of hepatocellular carcinoma. J Cell Biochem. 2018;119(11):94199432. doi: $10.1002 /$ jcb. 27259.

[27] Mu X, Shi W, Xu Y, Xu C, Zhao T, Geng B, Yang J, Pan J, Hu S, Zhang C, Zhang J, Wang C, Shen J, Che Y, Liu Z, Lv Y, Wen H, You Q. Tumor-derived lactate induces M2 macrophage polarization via the activation of the ERK/STAT3 signaling pathway in breast cancer. Cell Cycle. 2018;17(4):428-438. 


\section{Figure 1}

CD14 and CD105 cells are observed in Gaint cell tumor by tissue staining.

X-Ray indicates the lesion area of three GCT patients are tibia, ilium and both terminal of the tibia and femur, respectively. (d) HE staining indicates that the neoplasm is consist of three categories cells: multinucleated giant cells, mononuclear histiocytic cells and GCTSCs. Scale bar $=50 \mu \mathrm{m} .(\mathrm{e}-\mathrm{g})$ Immunofluorescent staining exhibits the STRO-1+ ${ }^{+}$cells (green) have a different distribution with $\mathrm{CD} 14^{+}$cells (red), which means that they belong to two distinguished lineages. Scale bar $=10 \mu \mathrm{m}$. 
$\mathbf{a}$

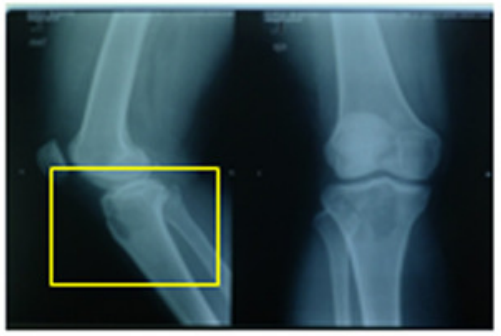

Female 46 years old

d

HE

CD14

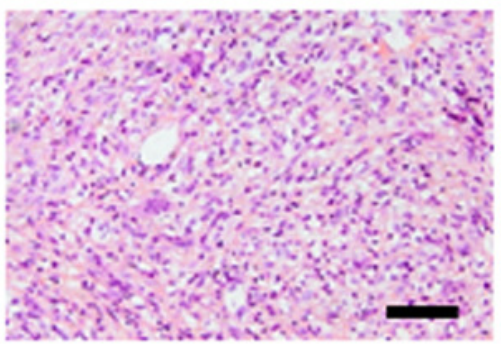

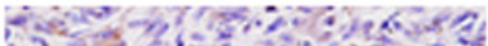
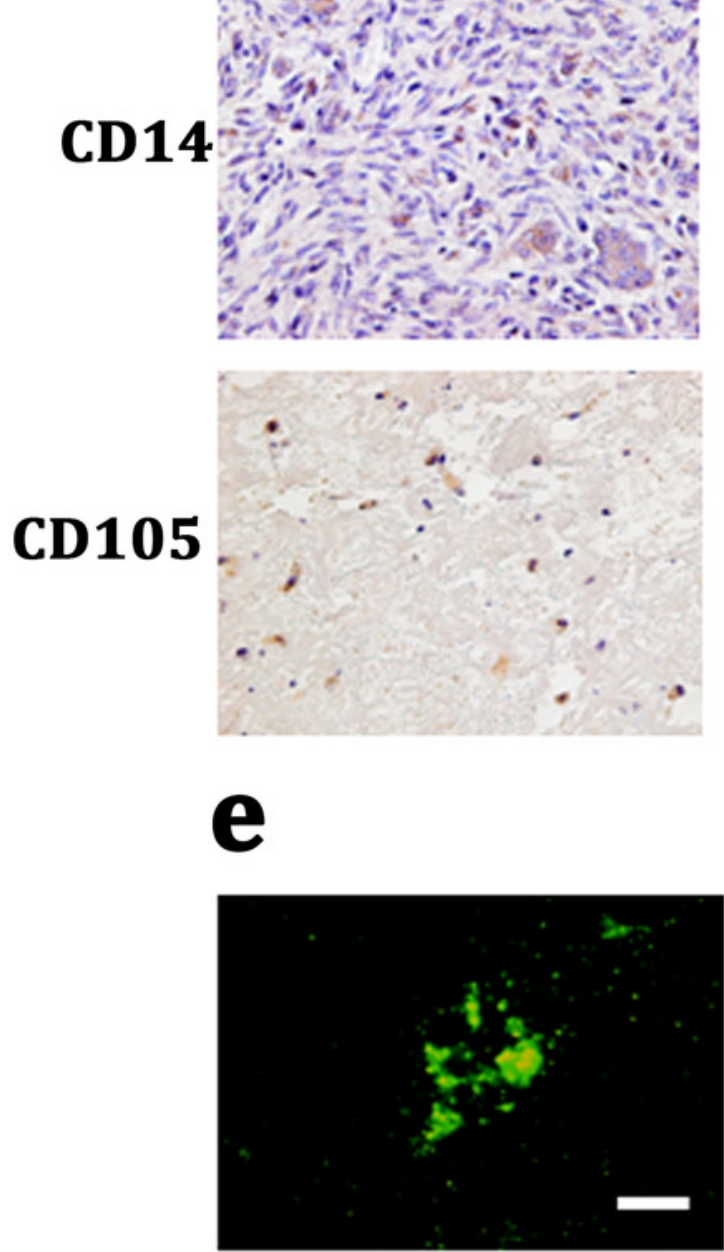

STR0-1
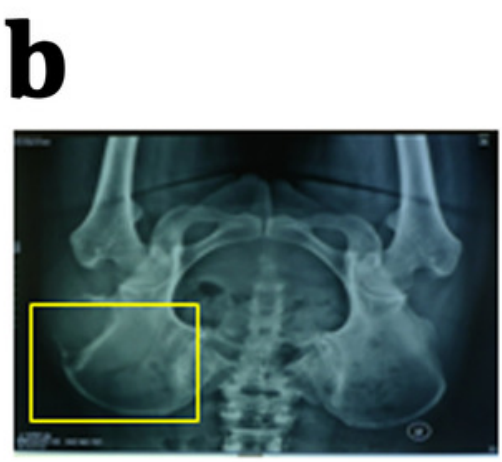

Female 41 years old
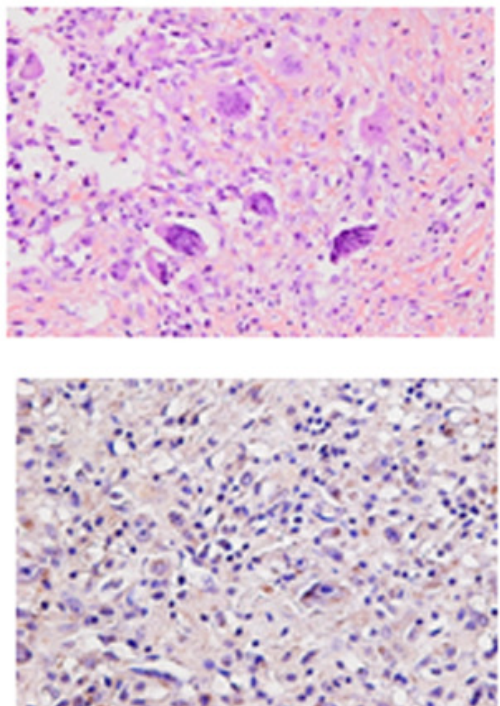

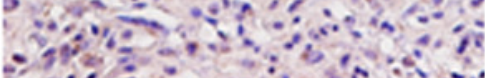
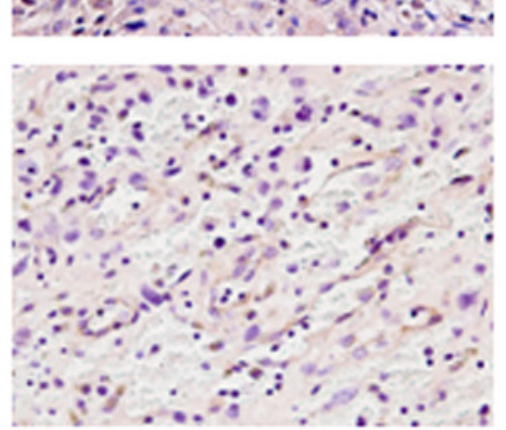

f

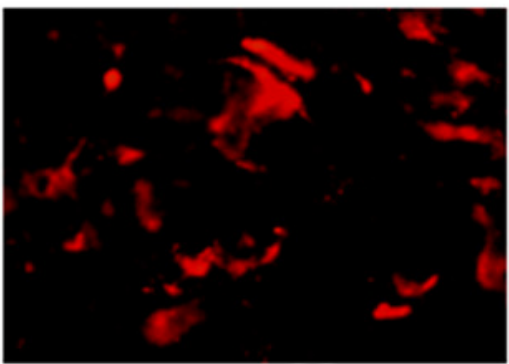

CD14

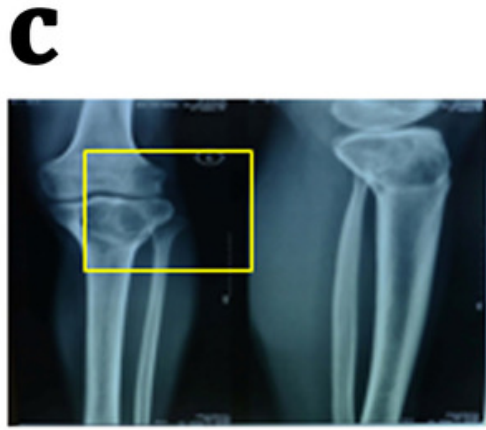

Male 21 years old
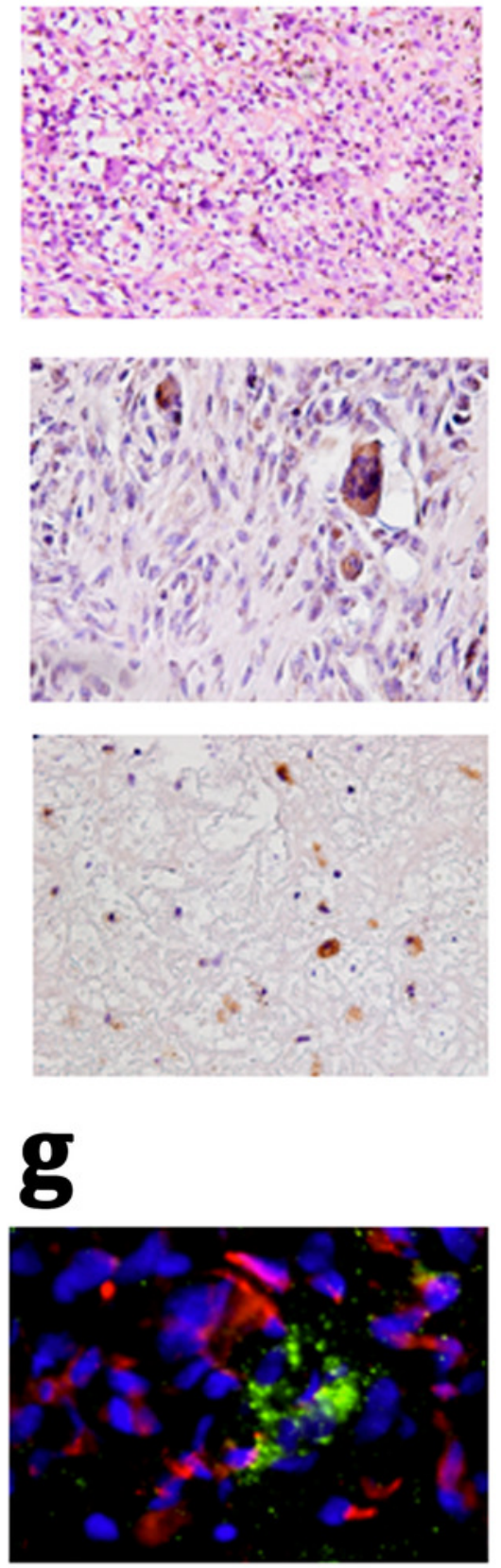

Merge 


\section{Figure 2}

GCTSCs showed increased proliferation and decreased differentiation characteristics.

(a) Flow cytometry analysis indicates that both of BMMSCs and GCTSC1, GCTSC2, GCTSC3 have common expressed MSCs markers CD90, CD105, CD29, and rarely express hematopoietic lineage markers CD14, CD34, CD45. (b, c) Proliferation ability of both BMMSCs and GCTSCs. Brdu measurement indicates the proliferating cells. Single-colonyderived MSCs and GCTSCs, respectively, were expanded to $2 \times 10^{6}$ cells and subsequently implanted into immunocompromised mice with the use of geofoams as a carrier. The proliferating cells in GCTSCS1 $(P<0.01)$ and GCTSCs2 $(P<0.05)$ are much more than BMMSCs. However, the GCTSCs3 after implantation shows a similar proliferation ability. (d, e) Osteogenic and adipogenic differentiation of BMMSCs and GCTSCs in vitro. (f) de novo bone formation of BMMSCs and GCTSCs in vivo. 
a
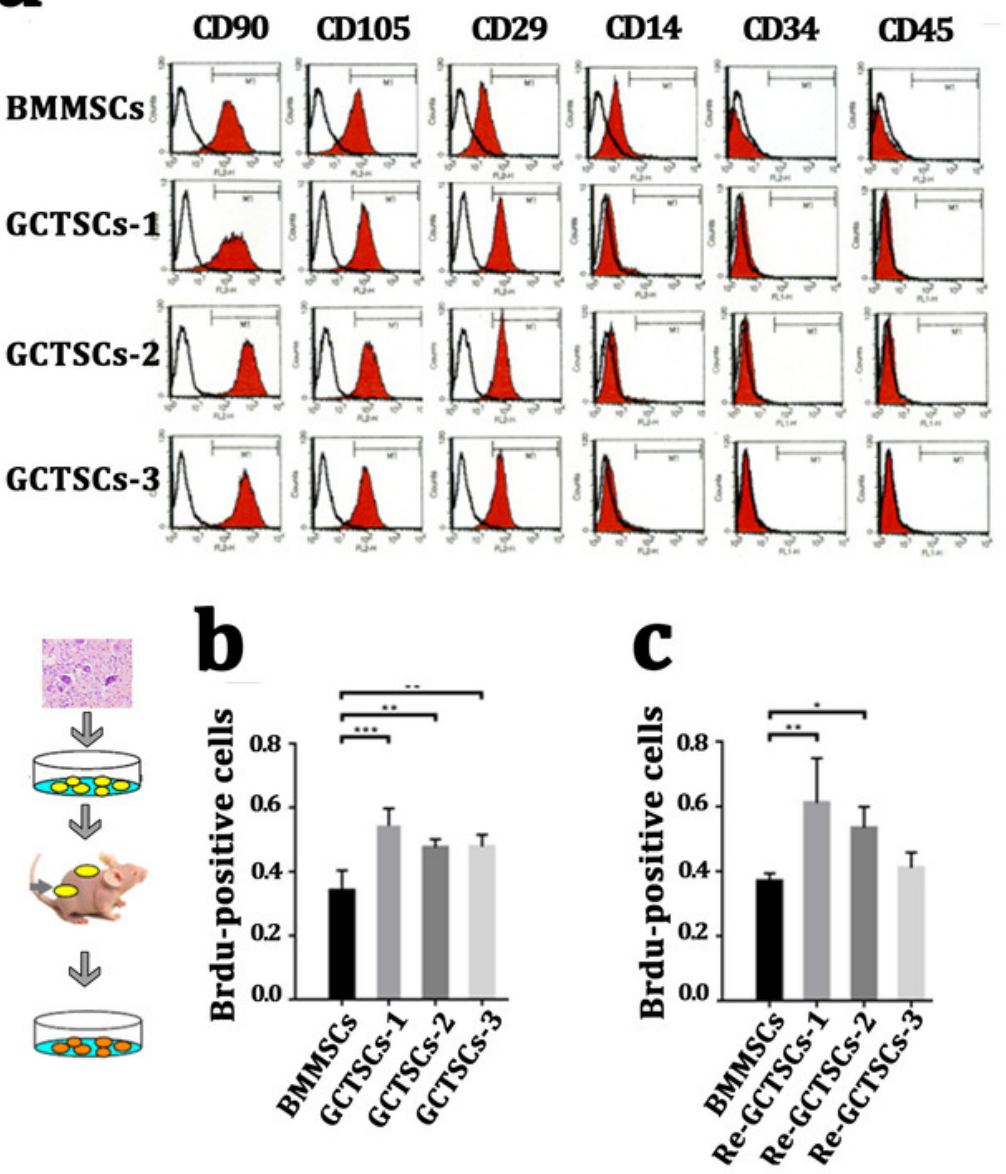

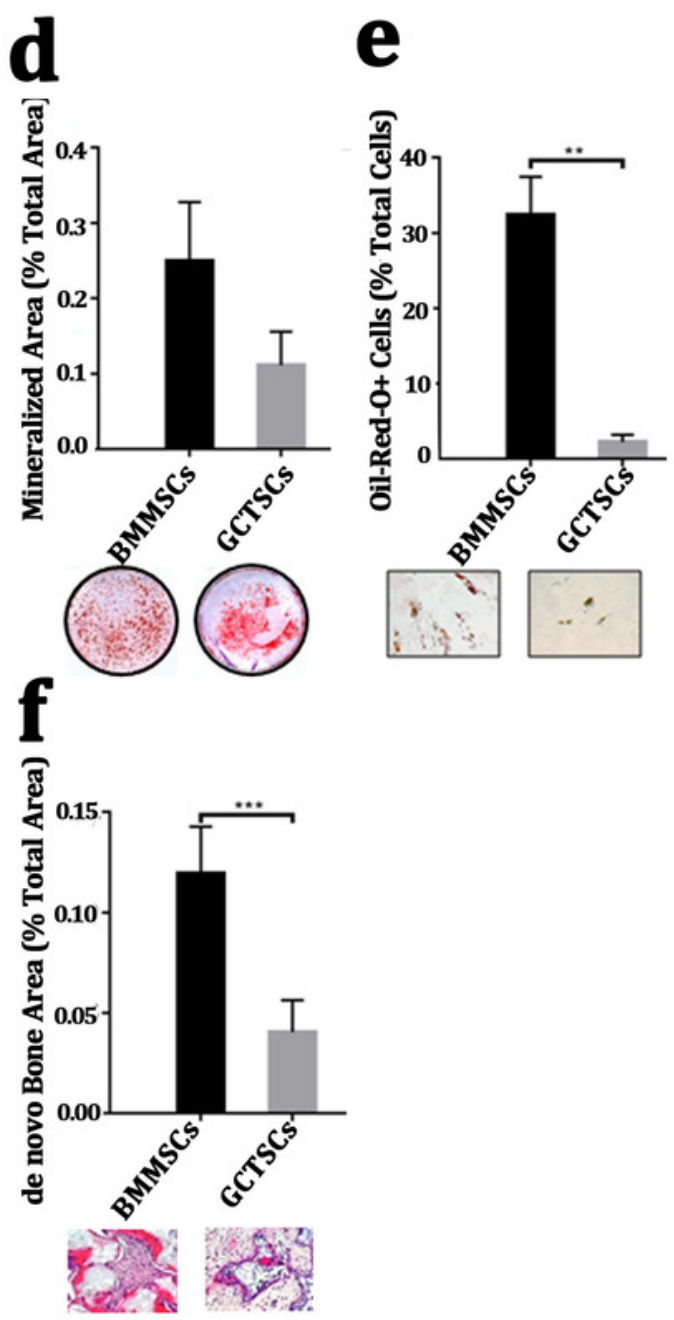




\section{Figure 3}

GCTSCs play no role in proliferation or apoptosis of CD14 cells.

(a) The macrophages number co-cultured with BMMSCs and GCTSCs were compared in 7, 14 and 21 days. It shows that the both BMMSCs and GCTSCs have a rare effect on macrophages cells number. (b) Ki67 expression in macrophages co-culturing with different types of cells shows a similar level $(P>0.05)$, which indicates that both BMMSCs and GCTSCs don't have a promotion for macrophages proliferation. (c) The apoptosis of macrophages co-culturing with different types of cells shows that BMMSCs $(P<0.001)$ have a prominent tendence to induce macrophages apoptosis, compared with GCTSCs $(P>0.05)$. (d) Representative flow cytometry analysis of macrophages proliferation and apoptosis co-cultured with BMMSCs and GCTSCs. 

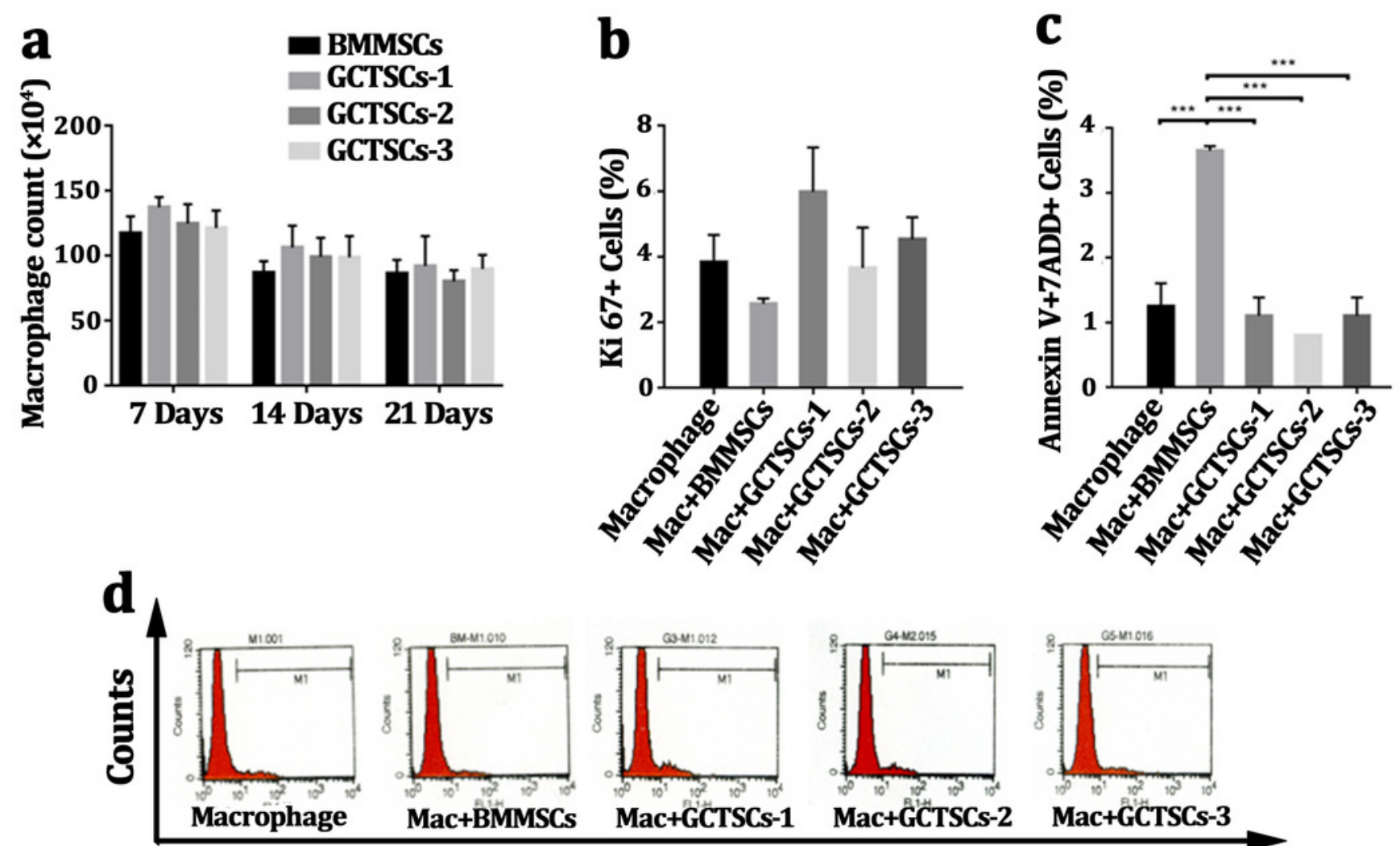

FL1-H

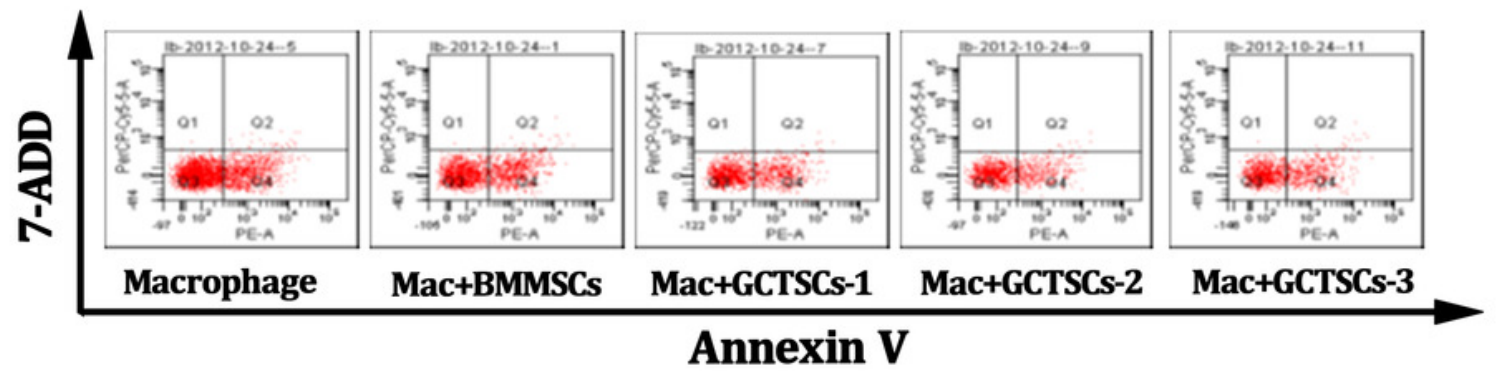




\section{Figure 4}

GCTSCs promote the polarization into M2 phenotype of macrophage via increasing IL- 6 and IL-10 release.

(a) The proportion of $\mathrm{CD} 206^{+}$cells among $\mathrm{CD} 14^{+}$cells. $\mathrm{CD} 206$ is a marker of $\mathrm{M} 2$ macrophages and it consists $85.05 \%$ of macrophages in GCT compared with $61.15 \%$ in blood $(P<0.05)$ $\mathrm{CD}_{206^{+}}$cells proportion in GCT CD14 ${ }^{+}$cells and co-cultured with BMMSC, GCTSC1, GCTSC2 and GCTSC3 (GCT CD14 ${ }^{+}$cell vs. GCT CD14 $4^{+}$cell + BMMSCs, $\mathrm{P}=0.483$. GCT CD14 ${ }^{+}$cell vs. GCT CD14 ${ }^{+}$cell + GCTSCs1, P=0.026. GCT CD14 ${ }^{+}$cell vs. GCT CD14 ${ }^{+}$cell $+\mathrm{GCTSCs2,} \mathrm{P}=0.044$. GCT $\mathrm{CD}_{14}{ }^{+}$cell vs. GCT CD14 ${ }^{+}$cell $+\mathrm{GCTSCs} 3, \mathrm{P}<0.001$ ). (c) Human cytokine antibody array analysis of BMMSCs and GCTSCs. Three independent experiments obtained a similar result and one of them are shown. The expression of IL- 6 and IL-10 of GCTSCs is much higher compared with BMMSCs (POS=Positive Control Spot, NEG=Negative Control Spot, BLANK=Blank Spot). (d) mRNA expression level of IL-6 and IL-10 among BMMSCs and GCTSCs. The expression of IL- 6 and IL-10 of GCTSCs is much higher compared with BMMSCs $\left({ }^{*} \mathrm{P}<0.05,{ }^{* * *} \mathrm{P}<0.001\right)$. (e) The proportion of $\mathrm{CD} 206^{+}$cells among THP-1 cells. 

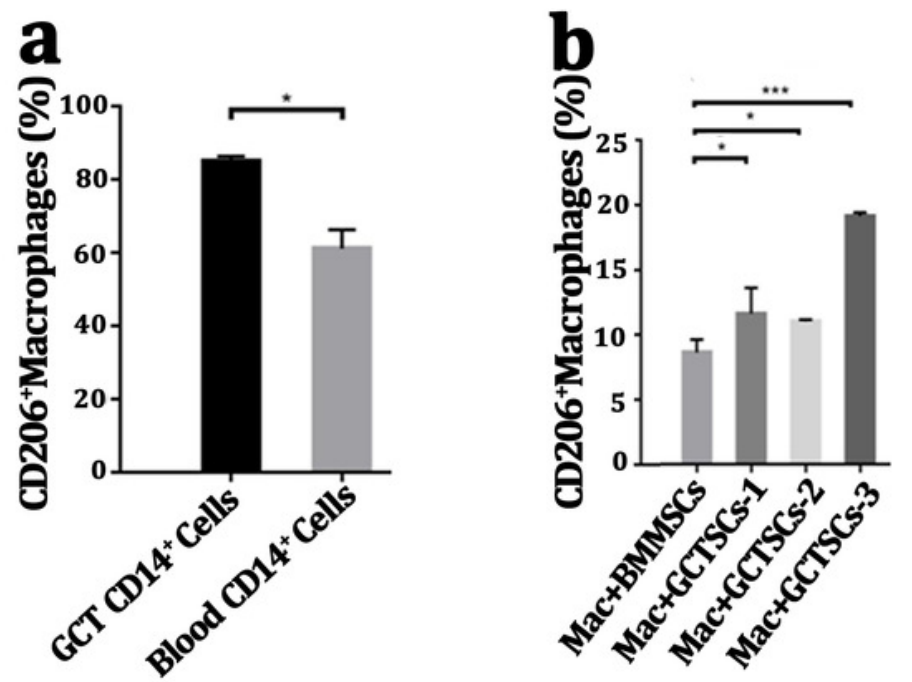

C
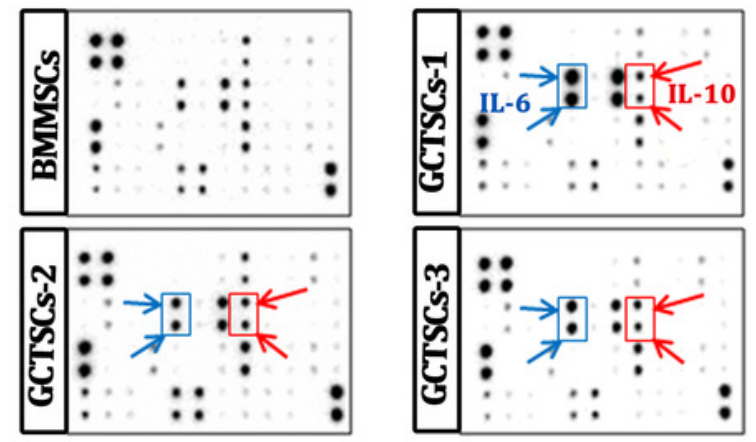

d
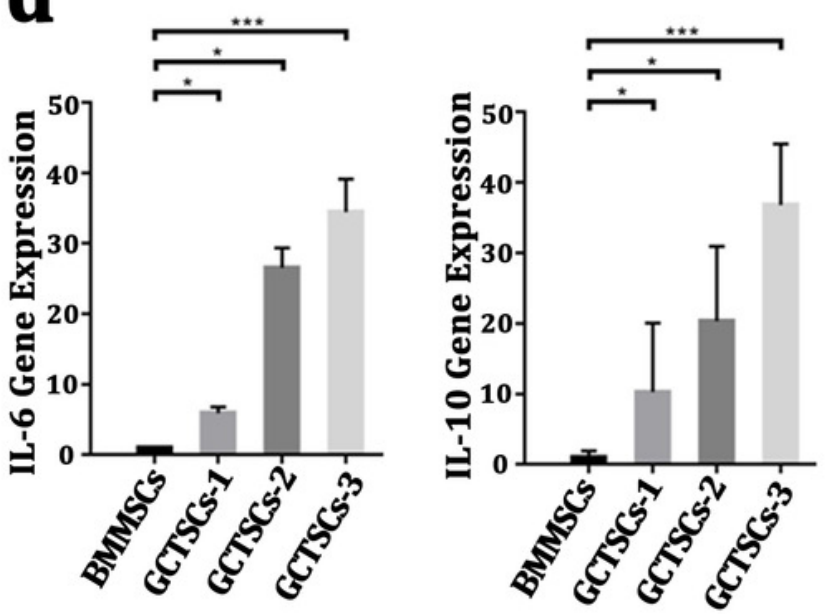

e

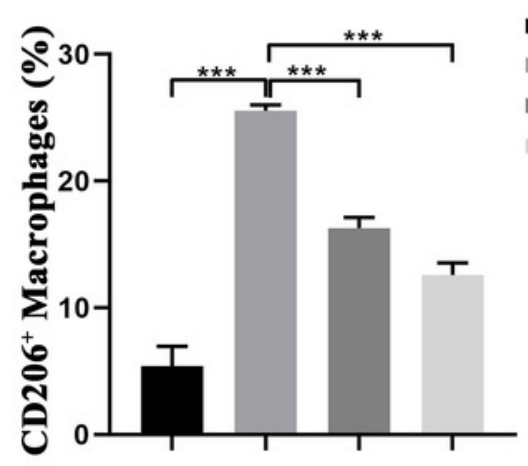

THP-1

- THP-1+GCTSCs ${ }^{\text {Ctr siRNA }}$

- THP-1+GCTSCs ${ }^{\text {I- }-6 \text { siRNA }}$

THP-1+GCTSCs ${ }^{\text {IL-10 siRNA }}$ 
Figure 5

Schematic diagram of this study.

Schematic illustration of GCTSCs exist in giant cell tumor, and release IL-6 and IL-10 to promote macrophages turning into M2 polarization phenotype.

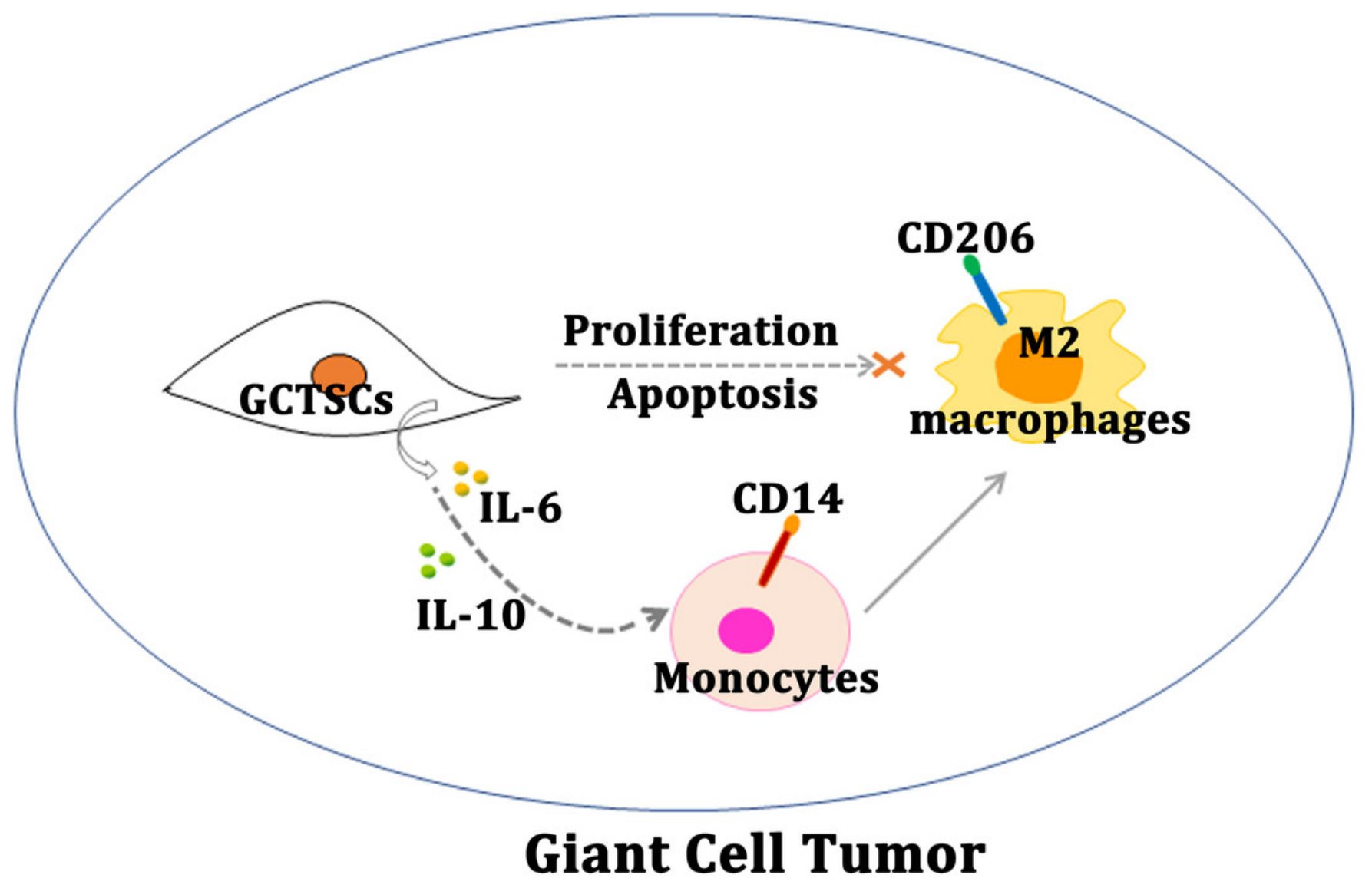

